\title{
Adubação nitrogenada e potássica no desenvolvimento de mudas de maracujazeiro
}

\author{
Nitrogen and potassic fertilization on development of passion fruit seedlings
}

\author{
Eliozéas Vicente Almeida ${ }^{1}$ William Natale ${ }^{2}$ Renato de Mello Prado ${ }^{3^{*}}$ José Carlos Barbosa $^{4}$
}

\section{RESUMO}

Este trabalho objetivou avaliar os efeitos da adubação com $N$ e K no desenvolvimento de mudas de maracujazeiro-amarelo (Passiflora edulis) cultivadas em Latossolo Vermelho distrófico. O delineamento experimental foi o inteiramente casualizado, com três repetições, num esquema fatorial $4 \times 4$, com 4 doses de $N$ (zero, 150, $300 e$ $600 \mathrm{mg}$ de $\mathrm{N} \mathrm{dm}^{-3}$ ) e quatro de $\mathrm{K}$ (zero, 75, 150 e 300mg de $\mathrm{K}$ $\left.d m^{-3}\right)$, parceladas em quatro vezes. A unidade experimental foi constituída por vasos de 3,3L. Após 84 dias da germinação, foram avaliados: altura da planta, o diâmetro do caule, o número de folhas e a área foliar. $O$ melhor desenvolvimento das mudas de maracujazeiro ocorreu com as doses de $150 \mathrm{mg}$ de $\mathrm{N} \mathrm{dm}^{-3}$ e de $300 \mathrm{mg}$ de $\mathrm{K} \mathrm{dm}^{-3}$.

Palavras-chave: nitrogênio, potássio, nutrição, maracujá, Passiflora edulis.

\section{ABSTRACT}

The present work aimed to evaluate the effects of nitrogen and potassic fertilization on the development and nutrition of yellow passion fruit (Passiflora edulis) seedlings, cultivated in dystrophic Red Latosol (Haplustox). The experimental design was completely randomized with 3 replications, in a $4 \times 4$ factorial design, between 4 levels of nitrogen (zero, 75, 150, 300 and 600 $\mathrm{mg} \mathrm{N} \mathrm{dm}^{-3}$ ) and 4 levels of potassium (zero, 75, 150 and 300mg $\mathrm{K} \mathrm{dm}^{-3}$ ). Fertilizers were divided into four parcels. The experimental unit consisted of pots of 3.3L. After 84 days of the germination, the plant height, diameter of the stem, number of leaves and leaf area were evaluated. The best development of seedlings was observed at the levels of $150 \mathrm{mg}$ of $\mathrm{N} \mathrm{dm}^{-3}$ and $300 \mathrm{mg}$ of $\mathrm{K} \mathrm{dm}^{-3}$.

Key words: nitrogen, potassium, nutrition, passion fruit, Passiflora edulis.

\section{INTRODUÇÃO}

No Brasil, um dos trabalhos pioneiros sobre a nutrição mineral do maracujazeiro-amarelo (Passiflora edulis) foi desenvolvido por HAAG et al. (1973), que verificaram que o nitrogênio e o potássio foram os nutrientes mais extraídos pelo maracujazeiro em condições de campo, aos 370 dias após o transplante. Trabalhos que avaliaram a resposta do maracujazeiro à adubação com N e K (MULLER et al., 1979; SOUZA et al., 1979) e NPK (COLAUTO et al.,1986; FARIA et al., 1991) não observaram efeitos dos fertilizantes na produção da cultura, em condições de campo.

Mudas de maracujazeiro cultivadas em solução nutritiva, com omissão de $\mathrm{N}$ ou mesmo com níveis baixos do nutriente, apresentaram diminuição no seu desenvolvimento (BLONDEAU \& BERTIN, 1978; Primavesi \& Malavolta, 1980). Trabalhos têm indicado também resposta positiva de mudas de maracujazeiro à adubação com N e K em relação à produção de matéria seca (LOPES et al., 1997). PRADO et al. (2004) trabalharam com adubação potássica, na forma de cloreto de potássio, com dose fixa de

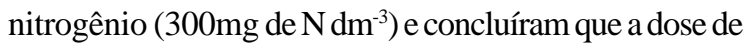
$225 \mathrm{mg}$ de $\mathrm{K} \mathrm{dm}^{-3}$ resultou em maior desenvolvimento das mudas, além de melhor nutrição e maior produção de matéria seca.

A resposta das culturas à adubação pode ser mais dependente da interação entre nutrientes do

\footnotetext{
${ }^{1}$ Departamento de Fitossanidade, Engenharia Rural e Solos, Faculdade de Engenharia, Universidade Estadual Paulista (Unesp), CP 31, 15385-000, Ilha Solteira, SP, Brasil. E-mail: eliozeas@zipmail.com.br.

${ }^{2}$ Departamento de Solos e Adubos, Faculdade de Ciências Agrárias e Veterinárias (FCAV), Unesp. Via de Acesso Prof. Paulo Donato Castellane, s/n, 14870-000, Jaboticabal, SP, Brasil. E-mail: natale@fcav.unesp.br.

${ }^{3}$ Departamento de Solos e Adubos, FCAV, Unesp. Jaboticabal, SP, Brasil. E-mail: rmprado@fcav.unesp.br. Autor para correspondência ${ }^{4}$ Departamento de Ciências Exatas, FCAV, Unesp. Jaboticabal, SP, Brasil. E-mail: jcbarbosa@fcav.unesp.br.
} 
que do nutriente isolado. Assim, na literatura é muito conhecida a interação positiva entre $\mathrm{N}$ e $\mathrm{K}$ (MALAVOLTA et al., 1997). Diante disso, os trabalhos de adubação fatoriais NK são importantes, pois podem promover maior contribuição prática para o manejo racional da adubação em comparação a trabalhos com os nutrientes isolados.

Existe pouca informação na literatura sobre o uso adequado da adubação química na produção de mudas de maracujazeiro. Ressalva-se também que, mesmo entre os poucos experimentos com adubação, os resultados são conflitantes em relação à resposta do maracujazeiro aos fertilizantes nitrogenados e potássicos.

O presente trabalho objetivou avaliar os efeitos da adubação nitrogenada e potássica no desenvolvimento de mudas de maracujazeiro-amarelo (Passiflora edulis Sims f. flavicarpa Deg.) cultivadas em Latossolo Vermelho distrófico.

\section{MATERIAL E MÉTODOS}

O experimento foi conduzido na FCAV/ Unesp, em Jaboticabal-SP, situada à latitude de $21^{\circ} 15^{\prime}$ 22” S e longitude de $48^{\circ} 18^{\prime} 58^{\prime}$ W $\mathrm{W}$, com altitude média de 595m e clima mesotérmico com inverno seco, ou seja, tipo Cwa, conforme a classificação Köppen. Utilizou-se um Latossolo Vermelho distrófico (EMBRAPA, 1999) (camada 3-4m do perfil) como substrato para a produção das mudas de maracujazeiro, cujos atributos químicos para fins de fertilidade estão apresentados na tabela 1. Efetuaram-se as determinações analíticas de acordo com RAIJ et al. (2001).

Após a secagem da terra ao ar, este último foi passado em peneira com malha de $6 \mathrm{~mm}$ para destorroamento e, cerca de 30 dias antes da semeadura, realizou-se a aplicação de corretivo de acidez, com o objetivo de elevar o V a 80\% (PIZA JÚNIOR et al., 1996). Utilizou-se calcário calcinado $\left(\mathrm{CaO}=420 \mathrm{~g} \mathrm{~kg}^{-1}\right.$; $\mathrm{MgO}=250 \mathrm{~g} \mathrm{~kg}^{-1}$ e PRNT = 131\%). A dose de calcário calculada $\left(1,385 \mathrm{~g} \mathrm{vaso}^{-1}\right.$, em torno de $\left.0,9 \mathrm{t} \mathrm{ha}^{-1}\right)$ foi homogeneamente aplicada ao substrato, mantendo-se a umidade em torno de $70 \%$ da capacidade de campo, durante 30 dias, estando os resultados da análise química, após a calagem, apresentados na tabela 1 .

O delineamento experimental adotado foi o inteiramente casualizado, com 16 tratamentos e três repetições, em um esquema fatorial $4 \times 4$, sendo quatro doses de nitrogênio e quatro de potássio. Os tratamentos consistiram de doses crescentes de nitrogênio e de potássio, nas formas de nitrato de amônio (34\% de N) e cloreto de potássio (60\% de $\mathrm{K}_{2} \mathrm{O}$ ), calculadas tomando-se por base a recomendação geral de MALAVOLTA(1980), que indica o uso de 300mg de $\mathrm{N} \mathrm{dm}^{-3}$ e $150 \mathrm{mg}$ de $\mathrm{K} \mathrm{dm}^{-3}$ de solo para ensaio em vasos. As doses utilizadas foram: zero; 150; 300 e 600mg de N

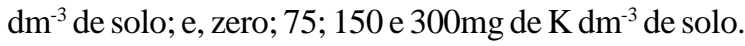

A unidade experimental foi constituída por vaso de alumínio com capacidade para 3,3 litros, empregando-se $3 \mathrm{dm}^{3}$ de substrato por vaso. $\mathrm{Na}$ semeadura, cada vaso recebeu doses de nivelamento de $\mathrm{P}$ (450 $\left.\mathrm{mg} \mathrm{dm}^{-3}\right)$, conforme indicação de MACHADO (1998), B (0,5mg dm $\left.{ }^{-3}\right)$, de acordo com a recomendação de MALAVOLTA (1980) e de Zn (5mg dm $\left.{ }^{-3}\right)$, segundo LOPES (2000). Como fontes de P, B e Zn, utilizou-se superfosfato simples ( $18 \%$ de $\mathrm{P}_{2} \mathrm{O}_{5}$ ), ácido bórico (17\% de B) e sulfato de zinco ( $22 \%$ de Zn), respectivamente. As doses de nitrogênio e de potássio foram parceladas em quatro vezes, aplicando-se $20 \%$ na semeadura, $30 \%$ quinze dias após a emergência, 30\% trinta dias após a emergência e $20 \%$ quarenta e cinco dias após a emergência.

A semeadura foi realizada em 01-05-2003, utilizando-se oito sementes por vaso e, após a germinação, foi feito o desbaste, deixando-se duas plantas por recipiente. Durante o desenvolvimento das mudas, efetuaram-se a pesagem e o rodízio diário dos vasos, mantendo-se a umidade em torno de $70 \%$ da capacidade de campo do substrato. Estando as mudas suficientemente desenvolvidas para serem implantadas no campo, aos 84 dias após a germinação, foram avaliadas as características fitotécnicas altura, diâmetro do caule, número de folhas e área foliar. Em seguida, as plantas foram colhidas, separando-se a parte aérea e as raízes, estas separadas do solo através de lavagem; todo material vegetativo foi lavado e secado em estufa a $65^{\circ} \mathrm{C}$, determinando-se o peso da matéria seca (duas plantas vaso $^{-1}$ ).

Os dados obtidos foram submetidos à análise de variância. Os resultados, cujas médias apresentaram diferenças significativas, foram submetidos à análise de regressão polinomial. Quando a interação $\mathrm{N} x \mathrm{~K}$ foi significativa, foram estabelecidas superfícies de resposta.

\section{RESULTADOS E DISCUSSÃO}

Houve efeito significativo das doses de $\mathrm{Ne}$ $\mathrm{K}$ aplicadas para todos para todas as características, bem como avaliadas nas mudas de maracujazeiro, das doses de N e K aplicadas, bem como interação entre os 
Tabela 1 - Características químicas do Latossolo Vermelho distrófico utilizado como substrato ${ }^{(*)}$ na produção de mudas de maracujazeiro.

\begin{tabular}{|c|c|c|c|c|c|c|c|}
\hline $\mathrm{pH}\left(\mathrm{CaCl}_{2}\right)$ & M.O. & $\mathrm{P}$ (resina) & K & $\mathrm{Ca}$ & $\mathrm{Mg}$ & $\mathrm{H}+\mathrm{Al}$ & $\mathrm{V}$ \\
\hline \multicolumn{7}{|c|}{ 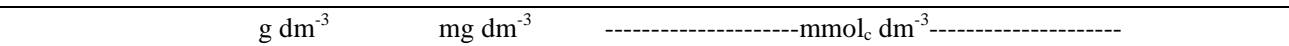 } & $\%$ \\
\hline \multicolumn{8}{|c|}{ Antes da calagem } \\
\hline 4,2 & 3 & 1 & 0,2 & 2 & 1 & 16 & 17 \\
\hline \multicolumn{8}{|c|}{ Após a calagem (semeadura) } \\
\hline 6,5 & 2 & 2 & 0,1 & 13 & 6 & 10 & 66 \\
\hline
\end{tabular}

(*) O substrato é resultado da mistura da camada de 3-4m do perfil do solo.

nutrientes para as características número de folhas e área foliar (Tabelas 2 e 3). A altura de plantas apresentou comportamento quadrático em resposta à adubação nitrogenada, atingindo o máximo desenvolvimento, pelo estudo da regressão, na dose próxima de 370mg de N $\mathrm{dm}^{-3}$. No entanto, pela comparação das médias (Tabela 2), como não houve diferença entre as doses 150, 300 e $600 \mathrm{mg} \mathrm{de} \mathrm{N} \mathrm{dm}^{-3}$, pode-se inferir que a dose de $150 \mathrm{mg}$ de $\mathrm{N} \mathrm{dm}{ }^{-3}$ é suficiente para o adequado desenvolvimento das mudas. Com relação ao potássio, a altura da planta apresentou, pelo estudo da regressão, comportamento linear, atingindo o máximo desenvolvimento na dose de 300 $\mathrm{mg}$ de $\mathrm{K} \mathrm{dm}^{-3}$. LOPES et al. (1997) também observaram efeitos positivos dos fertilizantes nitrogenados e potássicos sobre a altura de plantas de maracujazeiro-amarelo.

O diâmetro do caule apresentou comportamento quadrático em função dos fertilizantes aplicados, atingindo o máximo desenvolvimento nas doses aproximadas de 342mg de $\mathrm{N}$ e $207 \mathrm{mg}$ de $\mathrm{K} \mathrm{dm}^{-3}$ (Tabela 3).

Com relação ao número de folhas, houve interação entre a adubação nitrogenada e a potássica, cujo ponto máximo foi de 16 folhas para as doses próximas de $385 \mathrm{mg}$ de $\mathrm{N}$ e de $218 \mathrm{mg}$ de $\mathrm{K} \mathrm{dm}^{-3}$. Corroborando este resultado, PRADO et al. (2004) observaram que o incremento das doses de potássio interferiu positivamente no número de folhas de maracujazeiro-amarelo.

Para as doses de potássio, o máximo acúmulo de matéria seca da parte aérea ocorreu na dose de 300mg de $\mathrm{K} \mathrm{dm}^{-3}$ (Tabela 3). LOPES (1996) observou efeito positivo do nitrogênio sobre a matéria seca da parte aérea de mudas de maracujazeiro, enquanto PRADO et al. (2004) observaram efeito positivo do fertilizante potássico sobre a matéria seca desta planta.

Tabela 2 - Altura de plantas, diâmetro do caule, número de folhas, área foliar e matéria seca da parte aérea e de raízes das mudas de maracujazeiro submetidas à adubação com 4 doses de $\mathrm{N}$ e 4 doses de $\mathrm{K}$, em combinação fatorial.

\begin{tabular}{|c|c|c|c|c|c|c|}
\hline Adubação & $\begin{array}{l}\text { Altura } \\
(\mathrm{cm})\end{array}$ & $\begin{array}{l}\text { Diâmetro do } \\
\text { caule (mm) }\end{array}$ & Número de folhas & $\begin{array}{l}\text { Área foliar } \\
\left(\mathrm{cm}^{2} \text { vaso }^{-1}\right)\end{array}$ & $\begin{array}{c}\text { Matéria seca de } \\
\text { parte aérea }\end{array}$ & $\begin{array}{c}\text { Matéria seca de } \\
\text { raiz }\end{array}$ \\
\hline $\mathrm{N}, \mathrm{mg} \mathrm{dm}^{-3}$ & & & & & \multicolumn{2}{|c|}{ 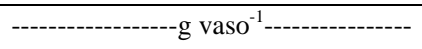 } \\
\hline 0 & $19,4 b$ & $2,7 b$ & $8 b$ & $463 b$ & $7,00 \mathrm{c}$ & $1,97 \mathrm{c}$ \\
\hline 150 & $64,1 \mathrm{a}$ & $3,5 a$ & $15 a$ & $1229 a$ & 15,33a & $3,47 a$ \\
\hline 300 & 53,9a & 3,3a & $14 a$ & $1235 a$ & 13,71ab & 3,17ab \\
\hline 600 & $51,1 \mathrm{a}$ & $3,1 \mathrm{a}$ & $14 \mathrm{a}$ & $1210 a$ & $12,44 \mathrm{a}$ & $2,48 \mathrm{bc}$ \\
\hline \multicolumn{7}{|l|}{$\mathrm{K}, \mathrm{mg} \mathrm{dm}^{-3}$} \\
\hline 0 & $36,5 b$ & $2,8 b$ & $11 b$ & $808 c$ & $9,57 c$ & $2,34 b$ \\
\hline 75 & 44,3ab & $3,2 \mathrm{a}$ & 13ab & $972 b c$ & $11,55 b c$ & 2,85ab \\
\hline 150 & $51,9 a$ & 3,3a & $14 a$ & 1113ab & $13,35 a b$ & 2,79ab \\
\hline 300 & $55,8 \mathrm{a}$ & $3,3 a$ & $14 \mathrm{a}$ & $1243 a$ & $14,00 \mathrm{a}$ & $3,11 \mathrm{a}$ \\
\hline \multicolumn{7}{|l|}{ Teste F } \\
\hline $\mathrm{N}$ & $31,04 * *$ & $12,43^{* *}$ & $45,97 * *$ & $76,48 * *$ & $38,83^{* *}$ & $13,93 * *$ \\
\hline $\mathrm{K}$ & $6,16^{* *}$ & $8,13^{* *}$ & $5,29 * *$ & $18,38^{* *}$ & $11,83^{* *}$ & $3,12 *$ \\
\hline NxK & $1,43^{\mathrm{NS}}$ & $1,28^{\mathrm{NS}}$ & $4,19 * *$ & $3,27 * *$ & $1,96^{\mathrm{NS}}$ & $0,92^{\mathrm{NS}}$ \\
\hline CV \% & 12,0 & 10,3 & 12,0 & 14,6 & 16,6 & 22,7 \\
\hline
\end{tabular}

Médias seguidas da mesma letra dentro da mesma fonte de variação ( $\mathrm{N}$ ou $\mathrm{K}$ ) não diferem entre si pelo teste de Tukey, a $5 \%$ de probabilidade; dados de duas plantas por vaso.

Ciência Rural, v.36, n.4, jul-ago, 2006. 
Tabela 3 - Relação entre as variáveis independentes (doses de N e K) e as variáveis dependentes (altura, diâmetro do caule, matéria seca da parte aérea e das raízes, número de folhas e área foliar) e as respectivas doses em que os valores de Y atingiram seus pontos de máximo de desenvolvimento.

\begin{tabular}{|c|c|c|c|c|}
\hline Variáveis & Equações & $\mathrm{R}^{2}$ & $Y \max$ & $\mathrm{X} \max$ \\
\hline $\mathrm{Af}(\mathrm{N})$ & $y=24,39+0,2215 x-0,000299 x^{2}$ & $0,73^{* *}$ & $64,5 \mathrm{~cm}$ & $370 \mathrm{mg} \mathrm{dm}^{-3}$ \\
\hline A f $(\mathrm{K})$ & $y=38,81+0,0634 x$ & $0,89 *$ & $58,8 \mathrm{~cm}$ & $300 \mathrm{mg} \mathrm{dm}^{-3}$ \\
\hline $\operatorname{DC} \mathrm{f}(\mathrm{N})$ & $y=2,78+0,0041 x-0,000006 x^{2}$ & $0,77 * *$ & $3,5 \mathrm{~mm}$ & $342 \mathrm{mg} \mathrm{dm}^{-3}$ \\
\hline DC f (K) & $y=2,78+0,0062 x-0,000015 x^{2}$ & $0,98 *$ & $3,4 \mathrm{~mm}$ & $207 \mathrm{mg} \mathrm{dm}^{-3}$ \\
\hline MSPA f (N) & $y=7,87+0,0434 x-0,000060 x^{2}$ & $0,77 * *$ & 15,7 g vaso $^{-1}$ & $362 \mathrm{mg} \mathrm{dm}^{-3}$ \\
\hline MSPA f (K) & $y=10,23+0,0144 x$ & $0,86^{* *}$ & $14,6 \mathrm{~g}$ vaso $^{-1}$ & $300 \mathrm{mg} \mathrm{dm}^{-3}$ \\
\hline $\operatorname{MSR} f(N)$ & $y=2,12+0,0084 x-0,000013 x^{2}$ & $0,81^{* *}$ & $3,5 \mathrm{~g}$ vaso $^{-1}$ & $323 \mathrm{mg} \mathrm{dm}^{-3}$ \\
\hline MSR f (K) & $y=2,48+0,0022 x^{2}$ & $0,79 *$ & 3,1 gaso $^{-1}$ & $300 \mathrm{mg} \mathrm{dm}^{-3}$ \\
\hline NF f $(\mathrm{NxK})$ & $\begin{array}{l}\mathrm{y}=7,298469+0,035974 \mathrm{~N}+0,023110 \mathrm{~K}- \\
0,000045118 \mathrm{~N}^{2}-0,000005805 \mathrm{NxK}-0,000047813 \mathrm{~K}^{2}\end{array}$ & $0,69 *$ & 16 folhas & $\begin{array}{l}385 \mathrm{mg} \mathrm{N} \mathrm{dm}^{-3} \\
218 \mathrm{mg} \mathrm{K} \mathrm{dm}^{-3}\end{array}$ \\
\hline AF f (NxK) & $\begin{array}{l}\mathrm{y}=346,22531+4,253576 \mathrm{~N}+2,193318 \mathrm{~K}- \\
0,005560 \mathrm{~N}^{2}+0,001456 \mathrm{NxK}-0,003725 \mathrm{~K}^{2}\end{array}$ & $0,84 * *$ & $1681 \mathrm{~cm}^{2}$ & $\begin{array}{l}432 \mathrm{mg} \mathrm{N} \mathrm{dm}^{-3} \\
379 \mathrm{mg} \mathrm{N} \mathrm{dm}^{-3}\end{array}$ \\
\hline
\end{tabular}

*, ** Respectivamente: significativo a 5 e 1\% de probabilidade pelo Teste F. A=altura, DC=diâmetro do caule, MSPA=matéria seca da parte aérea, MSR=matéria seca de raízes, $\mathrm{NF}=$ número de folhas, $\mathrm{AF}=$ área foliar.

A produção de matéria seca da parte aérea, em função das doses de $\mathrm{N}$ aplicadas (ajustou-se os valores a uma equação quadrática) atingiu a máxima produção (16g vaso-1) na dose aproximada de 362mg de $\mathrm{N} \mathrm{dm}^{-3}$ (Figura 1). Entretanto, estabelecendo um nível crítico (85\% da produção máxima), observa-se que a dose de $\mathrm{N}$ seria de $160 \mathrm{mg} \mathrm{dm}^{-3}$. Salienta-se, ainda, que esta diferença na dose de $\mathrm{N}$ para atingir o nível crítico e a máxima produção deve-se ao baixo ajuste do modelo quadrático, embora seja significativo. De qualquer forma, este resultado, mesmo proveniente de planta jovem, está de acordo com QUAGGIO et al. (1996).

Para a matéria seca das raízes, houve resposta para a fertilização nitrogenada, com a equação

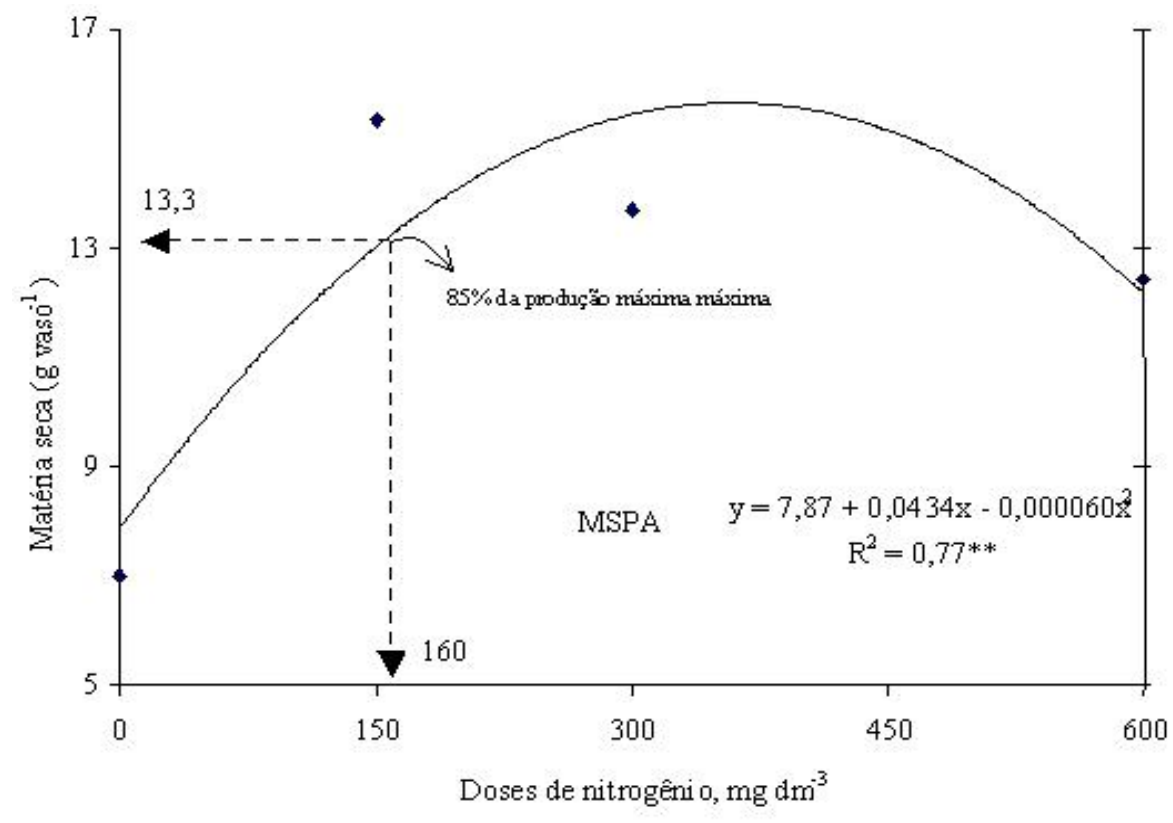

Figura 1 - Efeitos da aplicação de nitrogênio sobre a produção de matéria seca da parte aérea das mudas de maracujazeiro; dados de duas plantas por vaso.

Ciência Rural, v.36, n.4, jul-ago, 2006. 
ajustando-se ao modelo quadrático. O máximo acúmulo na dose aproximada de $323 \mathrm{mg} \mathrm{de} \mathrm{N} \mathrm{dm}^{-3}$, bem como de forma linear para a adubação potássica, com máximo acúmulo na dose de 300mg de $\mathrm{K} \mathrm{dm}^{-3}$ (Tabela 3). PEIXOTO \& PÁDUA (1989) e PRADO et al. (2004) verificaram efeitos positivos da adubação nitrogenada e da potássica, respectivamente, sobre a matéria seca das raízes de mudas de maracujazeiro.

\section{CONCLUSÃO}

O melhor desenvolvimento das mudas de maracujazeiro (altura, diâmetro do caule, número de folhas, área foliar e matéria seca de parte aérea e raiz) cultivadas em Latossolo Vermelho ocorreu com as doses de $150 \mathrm{mg}$ de $\mathrm{N} \mathrm{dm}^{-3}$ e de $300 \mathrm{mg}$ de $\mathrm{K} \mathrm{dm}^{-3}$, parceladas em quatro vezes.

\section{REFERÊNCIAS}

BLONDEAU, J.P.; BERTIN, Y. Carences minérales chez la grenadille (Passiflora edulis Sims var. flavicarpa) I. Carences totales en N, P, K, Ca, Mg. Crissance et symptômes. Fruits, Paris, v.33, n.6, p.433-443, 1978.

COLAUTO, N.M. et al. Efeito do nitrogênio, fósforo e potássio sobre a produção, qualidade e estado nutricional do maracujazeiro-amarelo. Pesquisa Agropecuária Brasileira, Brasília, v.21, n.7, p.691-695, 1986.

EMBRAPA. EMPRESA BRASILEIRA DE PESQUISA AGROPECUÁRIA. Centro Nacional de Pesquisa de Solo. Sistema Brasileiro de Classificação de Solos. Brasília: Produção de Informações, 1999. 412p.

FARIA, J.L.C. et al. Efecto de tres dosis de N, P y K en la producción de maracuyá amarillo (Passiflora edulis Sims. f. flavicarpa Deg.) durante tres años de evaluación en Guaíba, Rio Grande do Sul, Brasil. Pesquisa Agropecuária Brasileira, Brasília, v.26, n.3, p.311-314, 1991.

HAAG, H.P. et al. Absorção de nutrientes por duas variedades de maracujá. Anais da Escola Superior de Agricultura Luiz de Queiroz, Piracicaba, v.30, p.267-279, 1973.

LOPES, P.S.N. Propagação sexuada do maracujazeiroazedo (Passiflora edulis Sims. f. flavicarpa Deg.) em tubetes: Efeito da adubação nitrogenada e substrato. 1996. $52 \mathrm{f}$. Dissertação (Mestrado em Fitotecnia) - Universidade Federal de Lavras.
LOPES, P.S.N. et al. Efeito de nitrocálcio e cloreto de potássio sobre o desenvolvimento de mudas de maracujá amarelo propagadas em tubetes. Revista Brasileira de Fruticultura, Cruz das Almas, v.19, n.3, p.387-391, 1997.

LOPES, P.S.N. Micronutrientes em plantas juvenis de maracujazeiro doce (Passiflora alata Dryand). 2000. $111 \mathrm{f}$. Tese (Doutorado em Fitotecnia) - Universidade Federal de Lavras, Lavras.

MACHADO, R.A.F. Fósforo e zinco na nutrição e crescimento de mudas de maracujazeiro amarelo (Passiflora edulis Sims f. flavicarpa Deg). 1998. 93f. Dissertação (Mestrado em Fitotecnia) - Universidade Federal de Lavras, Lavras.

MALAVOLTA, E. Elementos da nutrição mineral de plantas. São Paulo: Ceres, 1980. 251p.

MALAVOLTA, E. et al. Avaliação do estado nutricional das plantas: princípios e aplicações. Piracicaba: POTAFOS, 1997. 319p.

MULLER, C.H. et al. Efeitos de doses de sulfato de amônio e de cloreto de potássio sobre a produtividade e qualidade de maracujás colhidos em épocas diferentes. Revista Ceres, Viçosa, v.26, n.143, p.48-64, 1979.

PEIXOTO, J.R.; PADUA, T. de. Efeito da matéria orgânica, do superfosfato simples e do cloreto de potássio na formação de mudas do maracujazeiro amarelo. Pesquisa Agropecuária Brasileira, Brasília, v.24, n.4, p.417-422, 1989.

PIZA JÚNIOR, C.T. et al. Adubação do maracujá. In: RAIJ, B. van et al. (Ed). Recomendações de adubação e calagem para o Estado de São Paulo. 2.ed. Campinas: IAC, 1996. Cap.17, p.148-149. (Boletim Técnico, 100).

PRADO, R.M. et al. Aplicação de potássio no estado nutricional e na produção de matéria seca de mudas de maracujazeiroamarelo. Revista Brasileira de Fruticultura, v.26, n.2, p.295-299, 2004.

PRIMAVESI, A.C.P.A.; MALAVOLTA, E. Estudos sobre a nutrição mineral do maracujá amarelo. VI. Efeito dos macronutrientes no desenvolvimento e composição mineral das plantas. Anais da Escola Superior de Agricultura Luiz de Queiroz, Piracicaba, v.37, n.2, p.609-630, 1980.

QUAGGIO, J.A. et al. Frutíferas. In: RAIJ, B. van; CANTARELLA, H. et al. (Ed). Recomendações de adubação e calagem para o Estado de São Paulo. 2.ed. Campinas: IAC, 1996. Cap.17, p.125. (Boletim Técnico, 100).

RAIJ, B. van et al. Análise química para avaliação da fertilidade do solo. Campinas: Instituto Agronômico, 2001. 285p. 